\title{
LA PERSPECTIVE FONCTIONNELLE DE LA PHRASE ET LA TYPOLOGIE
}

IVO VASILJEV

Prague

\begin{abstract}
En): This paper discusses the contributions of professor Vladimír Skalička and his disciples, especially professor Petr Sgall, towards the understanding of the functional sentence perspective from the angle of Vladimír Skalička's language typology. Based on their conclusions about the existence of an important fault line between the inflexional languages and all the other types of languages as far as the character and the interpretation of the so-called "free word order" is concerned, the author goes on showing how sentence constructions in Vietnamese, a predominantly polysynthetique language, can function in similar ways as word order does in the inflexional languages, when expressing the opposition of the theme and the rheme in various patterns of functional sentence perspective.
\end{abstract}

Keywords (En): deep structure; equivalence of meaning; functional sentence perspective; inflectional languages; language typology; rheme; surface structure; theme; Vietnamese; word order.

De toutes les typologies des langues, proposées par différents chercheurs, la typologie dont nous allons parler dans cette contribution est naturellement celle qui a été conçue par le professeur Vladimír Skalička au sein de l'école pragoise. Les traits essentiels de cette typologie ont été résumés par lui-même et son disciple et collègue Petr Sgall au commencement des années 1990, juste avant la mort de V. Skalička, de la façon suivante :

« Tout en acceptant les types bien connus, proposés jadis dans le cadre de l'ancienne classification morphologique des langues, la typologie structurale de Skalička ne repose plus sur une classification typologique stricte des langues. Elle ne retient de celle-ci qu'un certain nombre de traits. Désormais, les types ne représentent que des faisceaux de traits grammaticaux où la présence des uns tend à favoriser la présence simultanée des autres. Un type 'pur' n'est qu'un concept théorique (un construit) (SKALIČKA, 1966). Le système de toute langue naturelle représente toujours une combinaison de types différents. Ce qu'on peut dire tout au plus, c'est qu'il y a certaines langues dont la structure est marquée par un type dominant. Selon Vladimír Skalička et Petr Sgall, cette typologie possède une force explicatoire plus grande que celle d'autres modèles structuraux. Le but principal de cette typologie est une description et une analyse du rapport entre la structure grammaticale de surface et la structure profonde (ou sémantique). »

(SKALIČKA, SGALL, $1994: 343$ ).

Vladimír Skalička a donc postulé cinq types théoriques (ou construits typologiques) de langues, à savoir le type a) flexionnel, b) agglutinant, c) isolant ou analytique, d) polysynthétique et e) de flexion interne (SKALIČKA, 1966); dans certaines langues, chacun d'eux correspond à un type dominant, par exemple, a) le type flexionnel, en latin ou en tchèque, b) le type agglutinant, en hongrois ou en japonais, c) le type isolant ou analytique, en français ou en anglais, d) le type polysynthétique, en vietnamien ou en chinois, et finalement e) le type à flexion interne, en arabe. ${ }^{1}$

\footnotetext{
${ }^{1}$ Quiconque possède des connaissances minima sur la classification typologique (morphologique) traditionnelle des langues, se rendra compte que l'usage des termes « isolant » et « polysynthétique » chez Vladimir Skalička diffère de l'usage traditionnel et il est, en général, accepté par beaucoup de
} 
Où est donc la place de la perspective fonctionnelle de la phrase (ou la PFP) dans cette présentation? Pour pouvoir répondre à cette question, il faut d'abord préciser ce que l'on apelle, ici, la perspective fonctionnelle de la phrase.

Selon les linguistes de l'école pragoise, la perspective fonctionnelle de la phrase n'est, en effet, qu'un terme alternatif pour la segmentation de la phrase en thème et rhème (SVOBODA, 2002 : 149), cette dernière formulation étant préférée par le groupe du professeur P. Sgall, qui est, lui aussi, l'un des principaux protagonistes de la typologie pragoise, fondée et développée par Vladimír Skalička.

Une telle conception se base sur deux présuppositions importantes:

D'abord, le thème et le rhème font partie de la structure profonde (sémantique), caractéristique de toute langue humaine et relèvent des universaux.

Deuxièmement, le thème et le rhème, comme tous les autres éléments de la structure profonde, peuvent être exprimés, aux niveaux des structures de surface des différentes langues, par divers moyens grammaticaux.

En ce qui concerne la place de la perspective fonctionnelle de la phrase dans le cadre de la typologie linguistique pragoise, elle a été définie par Petr Sgall de la façon suivante :

«(...) la chose la plus intéressante du point de vue typologique, ce sont les relations qu'entretiennent la construction sémantique de la phrase et ses caractéristiques au niveau de la structure de surface. C'est ici qu'on trouve le plus souvent des 'faisceaux' de traits grammaticaux qui 's'impliquent les uns les autres' (comme, par exemple, la règle flexionnelle exigeant que chaque mot ait une seule désinence, d'un côté, et la liberté de l'ordre des mots, l'existence de la rection etc., de l'autre), ou bien l'expression isolante (analytique) des fonctions grammaticales par des mots spéciaux et l'absence de désinences et de rection... Il est évident que la segmentation de la phrase en thème et rhème doit tenir compte des moyens d'expression propres aux différentes langues, compte tenu de leurs caractéristique typologique.

On découvrira alors que l'une des différences principales est celle qui oppose, d'une part, les langues où l'ordre des mots est libre (ou, plus précisément, les langues où la place des éléments est avant tout déterminée par la segmentation de la phrase en thème-rhème ${ }^{2}$ ), et les autres langues. La plupart des langues slaves sont classées selon ces critères, tout comme le védique, le hittite, le grec, le latin et d'autres langues anciennes indo-européennes; on pourrait dire, en bref, que dans ces langues, l'ordre des mots est déterminé avant tout par la gradation de le dynamisme de l'expression [...]. En revanche, dans les langues isolantes (analytiques) de l'Europe Occidentale d'aujourd'hui, la segmentation thème-rhème est exprimée, plus ou moins, par divers moyens (ordre des mots, intonation, constructions grammaticales). Le troisième grand groupe est formé par les langues (surtout d'Asie Orientale, mais aussi d'Afrique) qui utilisent, pour la segmentation thème-rhème, des moyens morphologiques (dans la plupart des cas, des

linguistes. Skalička lui-même a expliqué sa conception du type polysynthétique, y compris les raisons pour lesquelles les langues de l'Amérique du Nord, traditionnellement considérées comme des langues prototypiques du type polysynthétique, ne le sont pas, en réalité, notamment dans son article sur les langues polysynthétiques (SKALIČKA, 1955). Cette explication a été reprise et commentée, plus récemment, par VYKYPĚL (2006 : 76-78 ) et VASILJEV (2009 : 94-97).

${ }^{2}$ Ajoutons encore une citation, où la même idée est rendue de façon encore plus claire: « [...] la différence fondamentale entre les langues, en ce qui concerne l'ordre des mots, réside dans la distinction entre l'ordre grammaticalisé et l'ordre 'libre', où l'ordre dit 'libre' n'est pas soumis aux restrictions émanant de la structure de surface; de ce fait, il se trouve déterminé de façon plus immédiate par les besoins d'exprimer le thème et le rhème. » (SKaličKa, SGall, 1994 : 346). L'ordre dit "grammaticalisé » est celui dont la fonction est d'exprimer les relations syntaxiques fondamentales, comme le sujet, l'objet, l'attribut, etc. 
particules polysynthétiques ou des affixes agglutinants). » (SGALL, 1982 : 177-178) - (souligné par I. V.). ${ }^{3}$

Il va sans dire que les langues du premier groupe sont les langues dominées par le type flexionnel, où :

«[...] nous observons une alternances de racines (éventuellement avec une seule désinence pour une forme lexicale donnée), ce qui implique un amalgame des fonctions dans les désinences (ces dernières expriment donc, simultanément le cas, le nombre et le genre ou la personne, le nombre, le temps et le mode) qui présentent un haut degré de synonymie et d'homonymie [...]; les adjectifs possèdent aussi des désinences qui expriment la rection grammaticale (en genre ou en un autre type de sous-catégorisation des substantifs); celle-ci, à son tour, permet que la perspective fonctionnelle de la phrase puisse être rendue par l'ordre de mots [...] » (SKALIČKA, SGALL, $1994: 348){ }^{4}$

La raison en est que l'ordre des mots dit «libre » est plus facilement réalisé par le fait que les fonctions syntaxiques de chaque substantif, adjectif ou verbe dans une langue dominée par le type flexionnel sont déjà marquées par la structure même des mots. Par conséquent, « le soi-disant ordre libre des mots est libre dans la mesure où il n'est pas dicté par des règles grammaticales de surface; il est donc déterminé, dans une plus ou moins grande mesure, par les fonctions de la structure profonde, destinées à exprimer le thème et le rhème. » (SKALIČKA, SGALL, 1994 : 349, remarque 6, se référant à SGALL, 1982). (Voir aussi la note 2, ci-dessus).

Par contre, dans les langues à dominante agglutinante, "l'ordre fixe des mots ne peut pas exprimer directement le thème et le rhème et les affixes n'y suffisent généralement pas non plus; la présence explicite d'une forme grammaticale exprimant la détermination y est donc plus nécessaire que dans les langues où une information 'donnée/connue' est visiblement présentée comme thème [grâce à sa position dans la phrase]. » (SKALIČKA, SGALL, 1994 : 350, remarque 15).

Il faut encore ajouter que depuis son article bien connu sur «La nécessité d'une linguistique de la parole », publié en 1948, le professeur Skalička ne cessait de souligner le rôle que la perspective fonctionnelle de la phrase joue spécifiquement au niveau de la syntaxe (SKALIČKA, 1948, 1960a, 1960b, 1961). Il concluait en disant que la phrase, en tant qu'unité de langue au sens saussurien, représente un phénomène plus complexe qui ne saurait se réduire à l'inventaire des règles syntaxiques, mais qui doit inclure également les principes de la perspective fonctionnelle. (SKALIČKA, 1948 : 33).

Le professeur Skalička a aussi souligné, à plusieurs reprises, qu'il existe des langues, comme le japonais, où la PFP peut être exprimée par des moyens morphologiques. Malheureusement, il ne mentionnait qu'un seul exemple, tiré d'un manuel de japonais : Nihon wa yama ga ooi (Japon - en ce qui concerne, montagne - nominatif, nombreux). (SKALIČKA, 1961, 1963 : 844). Néanmoins, ceci a, sans doute, encouragé certains de ses disciples, et en particulier Pavel Novák, à chercher la possibilité d'autres moyens pour exprimer la PFP dans certaines langues (NovÁK, 1958, 1974, republiés dans NovÁK, 2010 : 39, 122).

\footnotetext{
${ }^{3}$ Toutes les traductions de l'allemand, du tchèque, du vietnamien et du japonais en français ont été effectuées par l'auteur de cette communication.
} 
Nous pouvons ajouter qu'il manque des études systématiques et détaillées sur la perspective fonctionnelle de la phrase dans les langues du troisième groupe postulé par Petr Sgall, c'est-à-dire les langues d'Asie et d'Afrique, dans lesquelles le type agglutinant ou polysynthétique est souvent dominant. De plus, Petr Sgall ne se servait que de sources secondaires (voir LI, 1976 et KUNO, 1972).

Il nous semble, cependant, que les conclusions tirées par Vladimír Skalička et Petr Sgall sur l'ordre libre des mots dans les langues flexionnelles sont d'une telle importance qu'elles peuvent servir comme point de départ à de nouvelles réflexions.

Tout d'abord, si l'on compare les langues flexionnelles avec les langues polysynthétiques comme le vietnamien, on voit qu'un substantif, un verbe ou un adjectif d'une langue flexionnelle n'a pas le même comportement que les mots correspondants en vietnamien. Il est alors facile de reformuler la description des racines et des désinences données ci-dessus par Vladimír Skalička et Petr Sgall :

Prenons par exemple les mots d'une phrase tchèque: Seděli vedle nás starší manželé ('Il y avait un couple marié d'un certain âge assis près de nous'). Les mots dans cette phrase sont porteurs des informations suivantes :

Seděli: (sens lexical 'être assis' + verbe + intransitif + temps passé + aspect inaccompli + troisième personne + pluriel + genre masculin animé).

Vedle: (préposition, sens lexical 'à côté de')

Nás: (pronom de première personne 'nous' + pluriel + cas génitif, exigé par la préposition vedle)

Starši: (sens lexical 'âgé' + comparatif + nominatif)

Manželé: (sens lexical 'époux’ + substantif + pluriel + nominatif).

On peut dire qu'il s'agit là de mots qui sont porteurs des significations lexicales et grammaticales complexes, ce qui permet de codifier également la fonction syntaxique des différents éléments. Comme on l'a déjà constaté, c'est cette complexité des significations contenues dans des entités indissolubles qui rend ces unités libres, de sorte que l'ordre des mots dans la phrase peut être adapté à différentes situations ou contextes du moment et aux exigences de la personne qui parle. Ainsi on peut effectuer les permutations telles que :

\footnotetext{
Seděli vedle nás starší manželé. Rhème : un couple marié d'un certain âge

Starši manželé sedèli vedle nás. Rhème : à côté de nous

Starši manželé vedle nás seděli. Rhème : étaient assis
}

L'image de tels complexes peut être mise en relief de façon encore plus nette, si l'on examine quelques formes supplétives des pronoms personnels de la troisième personne au masculin singulier en tchèque :

on (lui / il) : nominatif

ho (lui, le) : accusatif, forme conjointe (donc thématique)

jeho (lui): accusatif, forme disjointe (donc rhématique) 
$m u$ (à lui, lui): datif, forme conjointe (donc thématique)

јетu (à lui): datif, forme disjointe (donc rhématique) ${ }^{5}$

Ces complexes de significations lexico-grammaticales ne peuvent pas être remplacés les uns par les autres dans leur position respective et sont aussi soumis à certaines limitations syntaxiques tout en étant des mots disposant d'une autonomie assez considérable ${ }^{6}$ (já [jsem] ho tam nikdy, jak je rok dlouhý, neviděl - 'je ne l'y ai jamais vu de toute l'année', la partie soulignée séparant l'auxiliaire facultatif 'jsem' et le pronom conjoint 'ho' d'une part, et le verbe lexicalement plein 'neviděl' de l'autre ; cf. neviděl jsem ho tam - 'je ne l'y ai pas vu'). Il est vrai que la complexité de ces unités lexico-grammaticales peut être mieux appréciée par des personnes dont la langue maternelle est une langue polysynthétique.

$\mathrm{Au}$ contraire, en vietnamien et dans d'autres langues polysynthétiques, les mots sont porteurs de significations lexicales pures et simples; leur forme n'indique même pas la partie du discours à laquelle ils appartiennent. Toutes les fonctions grammaticales y sont exprimées par des moyens qui se trouvent en dehors de ces mots. Prenons, par exemple, les mots suivants que nous allons revoir dans une phrase citée ci-dessous : con 'enfant', không 'ne pas exister', được 'recevoir', phép 'permission', đi 'aller', đá 'botter', bóng 'ballon'. Ces mots sont toujours porteurs d'un seul élement sémantique. En principe, leur place dans la phrase ne peut être changée sans changement ou destruction du sens de la phrase.

Il est néanmoins possible d'exprimer la perspective fonctionnelle de la phrase en changeant la position de certains groupes de mots qui représentent, eux aussi, des complexes lexico-grammaticaux, en l'occurrence, de type polysynthétique, dans lesquels toutes les fonctions grammaticales sont exprimées en dehors des mots - par la position des mots dans la phrase ou par des mots auxiliaires (grammaticaux), donc par des moyens polysynthétiques :

a. (Chúng con không được phép) (đi đá bóng) (marque du pluriel - enfant - ne pas exister - recevoir - permission - aller - botter - ballon) : 'On ne nous [enfants] a pas permis d'aller jouer au football'. Cet ordre des mots représente la PFP de base, plus ou moins indépendante du contexte.

b.(Đi đá bóng) là (chúng con không được phép): 'Aller jouer au football ne nous est pas permis'.

c.(Chúng con không được phép) là (đi đá bóng) (marque du pluriel - enfant ne pas exister - recevoir - permission - copule - aller - botter - ballon) : 'Ce qui ne nous est pas permis, c'est d'aller jouer au football'. (VASILJEV, 2009 : 126).

\footnotetext{
${ }^{5}$ On voit ici, d'ailleurs, un cas, où l'expression du rhème dans une langue flexionnelle se sert de moyens morphologiques (HAJIČOVÁ, $2002: 32$ ).

${ }^{6}$ Une définition générale du mot convenant aux différents types de langues qui se sert de la notion d'autonomie et de ses différents degrés, a été proposée par VASILJEV, 1969.
} 
Notons que le mot là dans les exemples (b) et (c) est une copule, un mot, donc un moyen grammatical polysynthétique, et qu'il est, en même temps, le seul élément qui fait la différence entre les exemples (a) et (c).

La limite entre le thème et le rhème en vietnamien peut aussi être marquée par un autre mot grammatical thì, comme dans le cas suivant: (Contexte: 'Tout le monde commettait des crimes'.) Nhung có một người thì khác - (Mais - il y a un - homme - particule thì - différent) - 'Mais un homme était différent'

Nous retrouvons, aussi, la même possibilité de déplacer certaines parties de la phrase, donc des complexes de significations lexico-grammaticales, pour exprimer la perspective fonctionnelle de la phrase dans des langues agglutinantes, comme en japonais ou en coréen, où la PFP peut être également marquée par des particules agglutinantes, donc par des moyens morphologiques, par exemple : jap. Kono hon wa ichiban takai desu (Ce - livre - particule de délimitation du thème le plus - cher - mot indicateur de politesse) et Ichiban takai no wa kono hon desu (où no représente un élément de substantivation).

On ne peut pas manquer de voir que le caractère des complexes lexicogrammaticaux dans certaines langues d'Asie Orientale est bien différent de celui des langues flexionnelles. D'un autre côté, il paraît être assez proche des moyens, dont se servent les langues dites analytiques (isolantes). On peut donc reconfirmer que la seule ligne de division importante entre les langues du point de vue de l'expression de la PFP au niveau de la structure de surface, est celle qui se situe entre les langues flexionnelles et toutes les autres.

Il est ainsi légitime de se poser la question de savoir quelles devraient être, désormais, les tâches de la typologie dans le domaine de la perspective fonctionnelle de la phrase. On verra tout de suite que cette question ne saurait être dissociée de celle de l'étude de la PFP en général.

Il est évident que le problème de la PFP ne peut pas être réduit à l'étude de l'ordre des mots. Il faut dire sans équivoque que l'articulation en thème - rhème est une fonction dans la structure profonde qui peut et doit être réalisée par différents moyens grammaticaux en surface. Ces derniers sont plus ou moins différents d'un type de langue à l'autre, voire même d'une langue à une autre. Ce que l'on a fait dans la plupart des cas, jusqu'à maintenant, c'est la description et l'explication plus ou moins détaillée des procédés de surface dans des langues particulières. Pour pouvoir comparer de façon satisfaisante les structures de surface des langues agglutinantes et polysynthétiques avec celles des langues flexionnelles et analytiques, il faut d'abord effectuer des études détaillées, telles que celles que nous venons de mentionner.

Mais cette tâche ne sera possible que si on a une base théorique assez bien définie. Ce n'est pas par hasard que Petr Sgall a demandé de trouver pour l'Explicandum, ce qu'est la PFP, un Explicatum dans le sens carnapien (SGALL, 1974 : 56) et que László Dezső et Györge Szépe ont constaté que «n’importe quelle théorie de la grammaire, si elle veut embrasser le sujet de la relation thème/rhème, doit être reformulée. » (DEZSÖ, SZÉPE, 1974 : 82). De manière analogue, Pavel Novák constate que «en réalité, on manque d'une théorie qui pourrait expliquer les phénomènes de la PFP. » (NOVÁK, 1974 : 175).

C'est sur la base d'un sentiment «quasi-unanime » des chercheurs que ces exigences et ces points de vue ont été formulés, il y a 38 ans. Or, il semble qu'ils 
soient encore valables de nos jours. D'ailleurs, le sujet vient d'être problématisé, encore récemment, par Bohumil VYKYPĚL (2006 : 55).

La raison pour laquelle une telle théorie est nécessaire est très simple et pratique : dans n'importe quelle comparaison des langues, surtout s'il s'agit de langues très différentes du point de vue typologique, l'un des points les plus difficiles est de trouver des significations équivalentes, d'autant qu'elles sont souvent cachées derrière des formes qui ne se ressemblent guère, ou ne se ressemblent pas du tout. Comment pourrait-on chercher et trouver des équivalences sans avoir une idée claire sur ce qu'on cherche? Ce sujet exigera une analyse particulière.

\section{BIBLIOGRAPHIE}

DEZsŐ László, SzÉPE György (1974), Two problems of Topic - Comment, in: DANEŠ F. (ed.) Papers on Functional Sentence Perspective. Prague, Academia, p. 81-96.

HAJIČOvá Eva (2002), Aktuální členění větné, in: P. KARLíK, M. NEKULA, J. PleSKAlOVÁ (eds.), Encyklopedický slovník češtiny, Praha, Lidové noviny, p. 32-34.

KUNO S. (1972), Functional Sentence Perspective, Linguistic Inquiry 3, p. 269320.

LI C. N. (ed., 1976), Subject and Topic, New York, Academic Press.

NovÁK Pavel (1958), K zdvojování předmětu v albánštině, in: K. HoRÁLEK, J. KURZ (eds.) Sborník slavistických prací věnovaných IV. Mezinárodnímu sjezdu slavistů v Moskvě, Prague, SPN, p. 27-32. Republié dans NovÁK (2010), p. 37-41.

NOVÁK Pavel (1974), Remarks on devices of functional sentence perspective, in: DANEŠ F. (ed.), Papers on Functional Sentence Perspective, Prague, Academia, p. 175-178. Republié dans NoVÁK (2010), p. 121-123.

NovÁK Pavel (2010), Lingvistika a jazyková realita. Výbor z díla, Praha, Akropolis.

SGALL Petr (1974), Zur Stellung der Thema-Rhema Gliederung in der Sprachbeschreibung, in: DANEŠ F. (ed.), Papers on Functional Sentence Perspective, Prague, Academia, p. 54-74.

SGALL Petr (1982), Zur Typologie der Thema-Rhema-Gliederung, in : Studien zum Tschechischen, Slowakischen und Deutschen aus vergleichender Sicht. Leipzig, p. 173-185.

SKALICKA Vladimír (1948), The Need for a Linguistics of La Parole, Recueil linguistique de Bratislava 1, p. 21-38.

SKALIČKA Vladimír (1955), Sur les langues polysynthétiques, Archiv orientální, 23, p. 10-28.

SKALIČKA Vladimír (1960a), Syntax promluvy (enunciace), Slovo a slovesnost 21, p. 241-249.

SKALIČKA Vladimír (1960b), Über die besonderen Formen der Syntax, in : Ruskočeské studie. Sborník Vysoké školy pedagogické v Praze, Jazyk a literatura, d. 2. Praha, VŠP, p. 37-42. 
SKALIČKA Vladimír (1961), Text, Kontext, Subtext, Acta Universitatis Carolinae, Philologica, Slavica Pragensia, p. 73-78.

SKALIČKA Vladimír (1963), Úvod do jazykovědy, in : Souborné dílo, 2. díl (1951 - 1963). Praha, Univerzita Karlova v Praze, Nakladatelství Karolinum, p. 825890.

SKALIČKA Vladimír (1966), Ein „typologisches Konstrukt“, in : VACHEK J. (ed.), Travaux linguistiques de Prague 2, Praha, Academia, p. 157-163.

SKALIČKA Vladimír, SGALL Petr (1984), Praguian typology of languages, in : LUELSDORFF Philip A. (ed.), The Prague School of structural and functional linguistics: a short introduction, Amsterdam, John Benjamins, p. 333-357.

SvoBodA Aleš (2002), Funkční perspektiva větná (FSP), in : P. KARLÍK, M. NeKUla, J. PlesKalová (eds.), Encyklopedický slovník češtiny, Praha, Lidové noviny, p. 149-150.

VASILJEV Ivo (1969), K typologické charakteristice slova (On Typological Characterization of the Word), Slovo a slovesnost, 30, p. 250-257.

VASILJEV Ivo (2009), The Type of Vietnamese, in : Bohumil VYKYPEL \& Vít BOCEK (eds.), Recherches fonctionnelles et structurales, Travaux linguistiques de Brno vol. 5, München, Lincom Europa, p. 93-131.

VYKYPĚL Bohumil (2006), Essais zur Prager Typologie (mit einer Bibliographia typologica Pragensis), LINCOM Studies in Language Typology 14, München, LINCOM EUROPA. 